\title{
Variação nictemeral na assembleia de peixes de um trecho de rio na Amazônia ocidental brasileira
}

\author{
Igor David da Costa ${ }^{*}$ \\ Wesclen Vilar Nogueira ${ }^{1}$ \\ Vanessa Martins da Rocha ${ }^{1}$ \\ Paulo de Tarso da Fonseca Albuquerque ${ }^{1}$ \\ Julia Myriam de Almeida Pereira ${ }^{1}$ \\ Rinaldo Antônio Ribeiro Filho ${ }^{2}$ \\ ${ }^{1}$ Fundação Universidade Federal de Rondônia, Campus Presidente Médici \\ Departamento de Engenharia de Pesca, Laboratório de Ciências Ambientais \\ Rua da Paz, 4376, CEP 76.916-000, Presidente Médici - RO, Brasil \\ ${ }^{2}$ Universidade Estadual Paulista Julio Mesquita Filho, Campus de Registro \\ Avenida Nelson Brihi Badur, 430, Vila Tupy, CEP 11.900-000, Registro - SP, Brasil \\ * Autor para correspondência \\ igorbiologia@yahoo.com.br
}

Submetido em $17 / 09 / 2014$

Aceito para publicação em 02/04/2015

\section{Resumo}

Padrões de atividade circadiana estão entre os ritmos biológicos mais evidentes e detectáveis nos animais. Este artigo tem por objetivo verificar os efeitos da variação nictemeral na abundância, biomassa e composição da ictiofauna no médio rio Machado, Rondônia. As coletas foram realizadas em nove pontos de amostragem no rio Machado, com utilização de malhadeiras. Foram capturados 1.320 espécimes, pertencentes a cinco ordens, 22 famílias e 56 espécies, com biomassa total de $689 \mathrm{~kg}$. Os Characiformes apresentaram maior abundância e biomassa em ambos os períodos; para o período noturno os Siluriformes foram o segundo grupo mais representativo em abundância. Para o período noturno, a biomassa dos Perciformes, Siluriformes, Clupeiformes e Gymnotiformes foram, na ordem, as superiores. Enfatizamos a ausência de segregação temporal no biótopo estudado, contudo, destacamos a contribuição em abundância das ordens Perciformes e Siluriformes na utilização do ambiente em períodos distintos dos citados na literatura. Todavia, apontamos que novas amostragens em ambos os períodos são necessárias por conta da ocorrência de um pequeno número de espécies raras exclusivas do período diurno $(\mathrm{S}=12 ; 21 \%)$ e noturno $(\mathrm{S}=8 ; 14 \%)$. Esses resultados são importantes para complementar os inventários taxonômicos anteriores à instalação de grandes empreendimentos ou áreas de proteção ambiental.

Palavras-chave: Biomassa; Ictiofauna; Rio Machado; Rondônia; Segregação temporal

\section{Abstract}

Nychthemeral variation in the fish assemblage at a river stretch in the western Brazilian Amazon. Circadian activity patterns are among the most obvious and detectable biological rhythms in animals. This article aims to check the effects of nychthemeral variation on abundance, biomass, and composition of the 
ichthyofauna in the middle Machado River, Rondônia, Brazil. Collection took place at 9 sampling sites in Machado River, by means of fishing nets. We captured 1,320 specimens, belonging to 5 orders, 22 families, and 56 species, and total biomass of $689 \mathrm{~kg}$. Characiformes had higher abundance and biomass in both periods; for nighttime, Siluriformes were the second most representative group in abundance. For nighttime, the biomass of Perciformes, Siluriformes, Clupeiformes, and Gymnotiformes were, in order, the higher. We emphasize the absence of temporal segregation in the biotope under study, but we highlight the contribution to abundance by the orders Perciformes and Siluriformes in using the environment at periods different from those cited in the literature. However, we point out that further sampling in both periods is required due to the occurrence of a small number of rare species unique to daytime $(\mathrm{S}=12,21 \%)$ and nighttime $(\mathrm{S}=8,14 \%)$. These results are significant to supplement the taxonomic inventories prior to the installation of large enterprises or environmental protection areas.

Key words: Biomass; Ichthyofauna; Machado river; Rondônia; Temporal segregation

\section{Introdução}

O ritmo circadiano está diretamente envolvido na organização temporal e espacial dos indivíduos e das comunidades, e na predição de resposta a eventos repetitivos (BOUJARD; LEATHERLAND, 1992). Segundo Volpato e Trajano (2006), os padrões de atividade circadiana estão entre os ritmos mais evidentes e detectáveis nos animais, constituindo o principal objeto de muitos estudos cronobiológicos de cunho natural e experimental. Ritmos diários de atividade são expressos através de padrões locomotores, associados a fases de alternância (período ativo vs. período de repouso), exploração de hábitat, alimentação e interações intra- e interespecíficas (formação de cardumes, comportamento agonístico, defesa de território, acasalamento e interações predador-presa) (VOLPATO; TRAJANO, 2006).

A importância dos padrões temporais de atividade de uma dada espécie esta relacionada ao ajuste de funções, tais como: estado fisiológico, atividades locomotoras e ciclo de vida, que com base nas mudanças temporais ocorrentes no ambiente podem aumentar ou diminuir as interações entre os indivíduos (reprodução, competição, relação predador-presa) (LAMPRECHT; WEBER, 1992). Diferenças interespecíficas na atividade constituem um importante fator envolvido na organização das comunidades de peixes, permitindo uma separação ecológica das assembleias de acordo com a fase do ciclo diário, quando cada uma das espécies concentra a maior parte das suas atividades (comportamento exploratório, alimentação, interações sociais, etc.) (VOLPATO; TRAJANO, 2006). Os peixes são classificados como noturnos (mais de $65 \%$ da atividade total durante a fase escura de um ciclo), diurnos (menos de $35 \%$ de atividade durante a fase escura do ciclo) e/ou crepusculares (e seus tipos mistos) que apresentam atividade entre $35 \%$ e $65 \%$ da fase com ausência de luminosidade (IIGO; TABATA, 1996; SÁNCHEZ-VÁZQUEZ et al., 1996).

A América do Sul contém a mais rica ictiofauna de água doce do mundo, porém, a avaliação e compreensão desta riqueza são negativamente afetadas pelo conhecimento incompleto de sua ecologia, biologia e sistemática (MENEZES, 1996). Estudos sobre a organização, estrutura, distribuição das comunidades e história natural de peixes são de suma importância, pois constituem informações úteis na gestão (MATTHEWS, 1998) e determinação de áreas de conservação.

A competição é supostamente uma das principais forças que dirigem a evolução da grande diversidade ictiofaunística da Amazônia, sendo provável que as diferentes formas e níveis de partilha de recursos sejam responsáveis pela manutenção desta diversidade ao longo do tempo (CARVALHO et al., 2007). Uma das formas de segregação ecológica entre as espécies é a temporal, onde o período de atividade das espécies é separado e regido pelo ciclo diário, resultando em grupos bem definidos de peixes diurnos e noturnos (ARRINGTON; WINEMILLER, 2003; PELICICE et al., 2005; SACCOL-PEREIRA; FIALHO, 2010; COSTA; FREITAS, 2010; DUARTE et al., 2012).

Para águas interiores neotropicais, a ictiofauna diurna é dominada por espécies de Characiformes e Cichlidae que se orientam visualmente (LOWE- 
MCCONNELL, 1964; 1987). A visão é empregada na formação de cardumes, durante as atividades de corte, para táticas de forrageamento e defesa (CARVALHO et al., 2007). Durante a noite, são encontrados em maior atividade espécies de grandes bagres e peixes elétricos que se movem principalmente próximo ao fundo. A baixa incidência, ou mesmo a ausência de luz obriga os peixes a empregarem outras formas de orientação para a percepção do ambiente, incluindo estímulos químicos, táteis, sonoros e elétricos. Esta segregação temporal resulta em um grande número de espécies que dividem o mesmo espaço e recursos alimentares (CARVALHO et al., 2007).

Entre os estudos ecológicos de assembleias de peixes neotropicais, poucos trabalhos têm caracterizado assembleias diurnas e noturnas em um único local (ARRINGTON; WINEMILLER, 2003; PELICICE et al., 2005; WILLIS et al., 2005; COSTA; FREITAS, 2010; DUARTE et al., 2010; 2012; COSTA et al., 2011).

O presente trabalho caracteriza a estrutura da assembleia de peixes em um trecho do rio Machado, um importante rio amazônico, e sua relação com a periodicidade diária. Baseado na premissa que Characiformes é o grupo mais abundante e rico em espécies, e apresenta maior atividade no período diurno, e Siluriformes, que são mais ativos durante o período noturno (LOWE-MCCONNELL, 1964; 1987) e representados por bagres de grande porte, nós testamos a hipótese que a mudança do fotoperíodo influencia a atividade dos peixes, resultando em mudanças na abundância, riqueza de espécies e biomassa da ictiofauna.

\section{Material e Métodos}

\section{Área de estudo}

O estudo foi realizado no rio Machado, situado na porção leste do estado de Rondônia. A bacia do rio Machado apresenta uma área de drenagem de 75.400 $\mathrm{km}^{2}$, descarga média anual de $700 \mathrm{~m}^{3} \cdot \mathrm{s}^{-1}$ e tem suas nascentes formadas por dois rios: Comemoração e Pimenta Bueno (KRUSCHE et al., 2005). O canal principal do rio tem comprimento total de $972 \mathrm{~km}$ e largura variando de 150 a $500 \mathrm{~m}$ (KRUSCHE et al., 2005). Com elevada presença de grandes rochas individualizadas, lajes rochosas (pedrais), troncos e galhos que são observados no período da vazante/seca (Figura 1). Segundo Goulding (1980) e Goulding et al. (2003), esse grande tributário do rio Madeira apresenta suas águas classificada como "clara", caracterizada por apresentar baixa carga de sedimento.

O clima da região é caracterizado por temperaturas que variam entre $19 \mathrm{e} 33^{\circ} \mathrm{C}$ e precipitação anual em torno de $2.500 \mathrm{~mm}$ (KRUSCHE et al., 2005), sendo o regime pluviométrico caracterizado por um período de seca (final de maio a setembro) e uma estação chuvosa (de outubro a abril) (FERNANDES; GUIMARÃES, 2002) com o pico da enchente em março e a cota mínima da vazante em setembro (CPRM, 2012). A reserva biológica do Jaru

FIGURA 1: Lajes rochosas, galhos (a) e corredeiras (b) no trecho médio do rio Machado (Área da Rebio Jaru) no período da vazante.
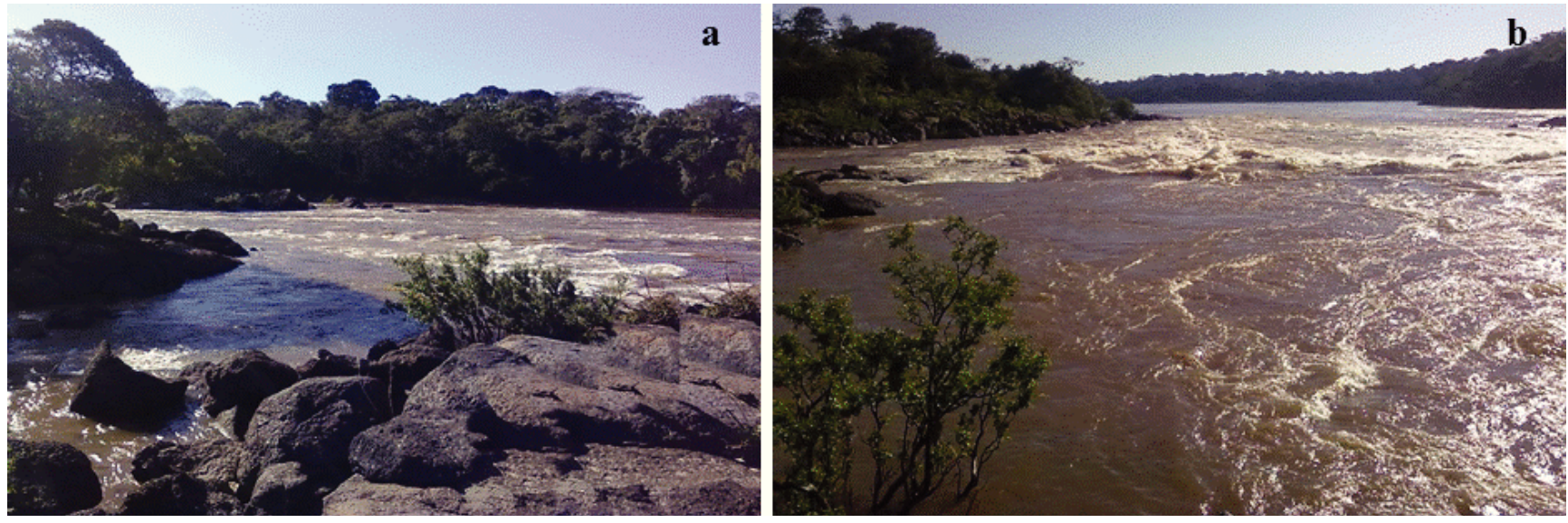
(ReBio) apresenta uma área total de $47.733 \mathrm{~km}^{2}$ (MMA, 2010) e localiza-se na bacia do rio Machado, bioma Amazônico. A região apresenta predominância de Floresta Ombrófila, principalmente aberta, com suas pequenas variações florísticas em função dos gradientes altitudinais e ripários, além de manchas de cerrado (IBGE, 1992).

\section{Amostragens}

As coletas foram realizadas nos seguintes pontos

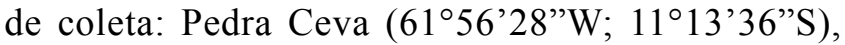

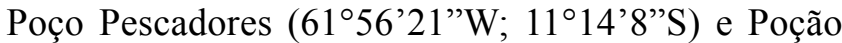
(6153'10”W; 1042'49”'S), situados fora dos limites da REBio Jaru; Cochabamba (61 ${ }^{\circ} 47^{\prime} 46^{\prime \prime} \mathrm{W}$; 09³1'55"S), Poço Pedra da Onça (61 ${ }^{\circ} 51^{\prime} 33^{\prime \prime}$ 'W; 0941'23”S), Retiro (6154'12”W; 0946'17’'S), Poço do Carmita (61 ${ }^{\circ} 54^{\prime} 50^{\prime \prime} \mathrm{W} ; 10^{\circ} 06^{\prime} 43$ 'S), Poço do Farofa (61 ${ }^{\circ} 54^{\prime} 28^{\prime \prime W}$; $10^{\circ} 10^{\prime} 52^{\prime \prime}$ 'S) e Poço São Sebastião (61 ${ }^{\circ} 50^{\prime} 44$ '”; $10^{\circ} 15^{\prime}$ '17'S) dentro dos limites da REBio Jaru (Figura 2), no período da vazante (julho e agosto) do ano de 2011, nos períodos de vazante, seca, enchente e cheia (fevereiro, março, junho a setembro e novembro)

FIGURA 2: Área de estudo com a indicação dos pontos de coleta no médio rio Machado-RO.

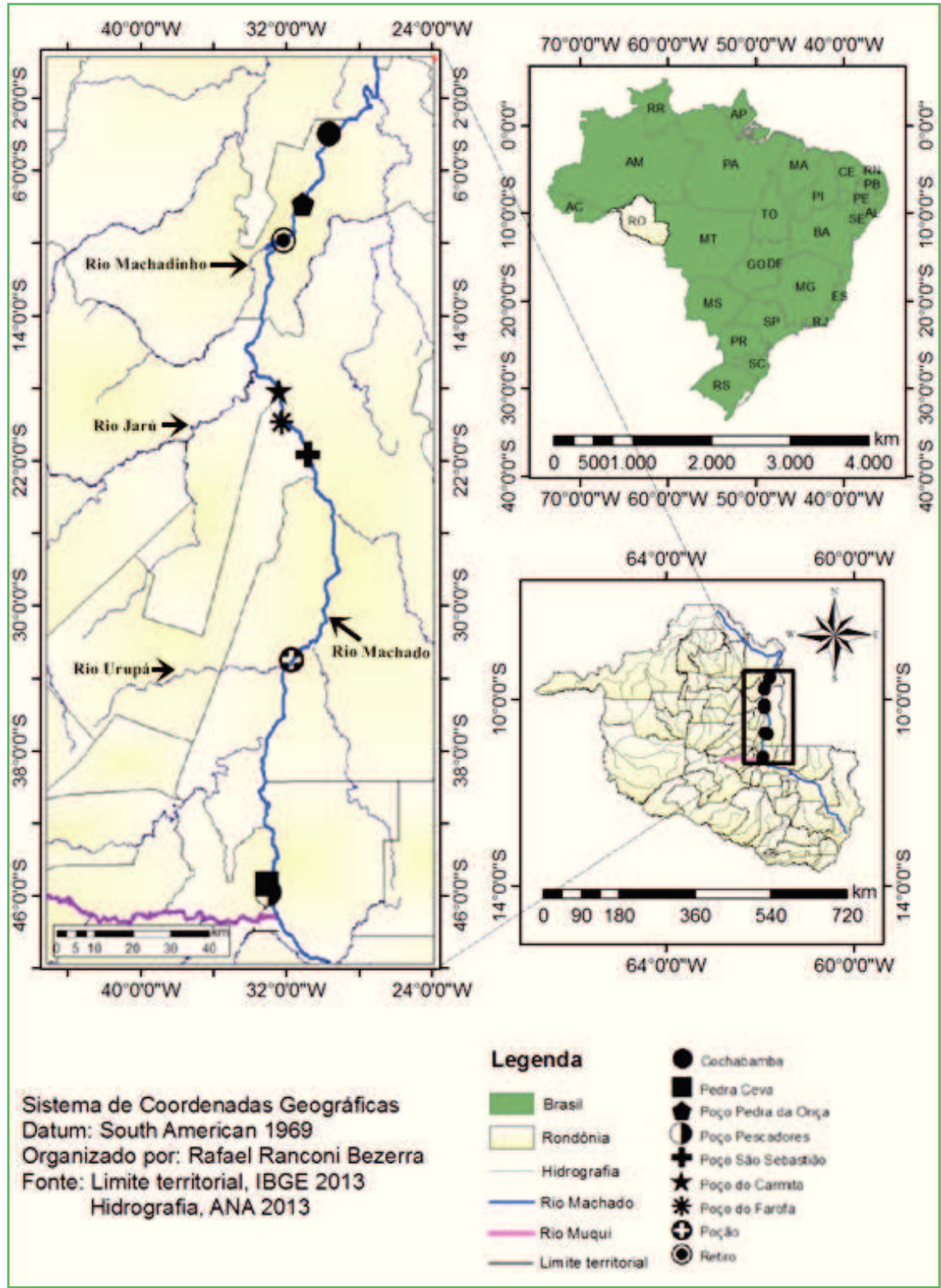


do ano de 2012 e enchente (julho e outubro) de 2013. Totalizando 73 amostras, 41 no período diurno e 32 no período noturno.

Para todas as coletas realizadas foram utilizadas 14 redes de emalhar com diversos tamanhos de malha entre nós opostos (mm) e dimensões (Tabela 1).

Todas as redes foram dispostas nas margens, porém no período de seca, estas foram localizadas na região central (calha) do rio. Estas permaneceram em cada ponto de coleta durante $24 \mathrm{~h}$, com despescas a cada $4 \mathrm{~h}$. Para as análises somente foram utilizadas as amostras oriundas do período diurno (07h00min-17h00min) e período noturno (19h00min-05h00min), não sendo utilizados os exemplares coletados nos períodos de crepúsculo matutino e vespertino.

TABELA 1: Dimensões das redes de emalhar utilizadas na coleta de peixes no médio do rio Machado/RO - Brasil. $\mathrm{C}=$ comprimento da rede e $\mathrm{A}=$ altura da rede.

\begin{tabular}{cccccc}
\hline $\begin{array}{c}\text { Malha } \\
(\mathbf{m m})\end{array}$ & $\begin{array}{c}\mathbf{C} \\
(\mathbf{m})\end{array}$ & $\begin{array}{c}\mathbf{A} \\
(\mathbf{m})\end{array}$ & $\begin{array}{c}\text { Malha } \\
(\mathbf{m m})\end{array}$ & $\begin{array}{c}\mathbf{C} \\
(\mathbf{m})\end{array}$ & $\begin{array}{c}\mathbf{A} \\
(\mathbf{m})\end{array}$ \\
\hline 20 & 2,20 & 1,20 & 80 & 5,20 & 1,45 \\
30 & 2,20 & 1,25 & 90 & 11,20 & 1,40 \\
35 & 2,30 & 1,45 & 100 & 13,00 & 1,40 \\
40 & 2,30 & 1,45 & 110 & 16,10 & 1,40 \\
50 & 2,50 & 1,80 & 120 & 16,10 & 1,70 \\
60 & 2,50 & 1,80 & 180 & 18,10 & 1,95 \\
70 & 3,20 & 1,45 & 200 & 18,10 & 2,00 \\
\hline
\end{tabular}

Os peixes capturados foram triados, identificados até o nível taxonômico mais preciso de acordo com Ferreira et al. (1998), Reis et al. (2003), Nelson (2006) e consultas aos pesquisadores da coleção ictiológica do Laboratório de Ictiologia e Pesca (LIP) da Universidade Federal de Rondônia - UNIR. Posteriormente, estes foram fixados em formol $10 \%$ e preservados em etanol a $70 \%$, sendo alguns exemplares depositados na coleção ictiológica da UNIR (código de tombamento: UFRO-ICT 023100, UFRO-ICT 023101, UFRO-ICT 023102, UFRO-ICT 023103, UFRO-ICT 023104, UFRO-ICT 023105, UFRO-ICT 023106 e UFRO-ICT 023107.

\section{Análise de dados}

Como análise exploratória foi realizado um Escalonamento Multidimensional não Métrico (EMnM) com os dados de abundância numérica e biomassa de cada espécie em cada período do dia (dia $v s$. noite) a fim de reduzir a dimensionalidade dos dados da assembleia de peixes. A matriz de dissimilaridade utilizada nesta ordenação foi construída utilizando o índice de BrayCurtis. Para tal análise foram excluídos os pontos de coleta com $n \leq 2$ indivíduos. O teste de Shapiro-Wilk de normalidade e o teste de Levene de homocedasticidade foram aplicados aos dados a fim de determinar a utilização de análises paramétricas (teste t pareado) ou não-paramétrica (U de Mann-Whitney) para comparação da riqueza de espécies, abundância e biomassa entre os períodos diurno e noturno.

Foi realizada uma análise de variância (ANOVA) bifatorial, utilizando modelos lineares gerais (GLM), sendo aplicado o teste de Tukey quando diferenças significativas para abundância e biomassa das espécies foram detectadas entre as médias (log 10). Como fontes de variação foram consideradas os períodos (dia e noite) e as ordens dos peixes (Characiformes, Siluriformes, Perciformes, Clupeiformes e Gymnotiformes).

Todos os dados foram previamente logtransformados $(\log x+1)$. O EMnM foi realizado com auxilio do software PAST 2.17 (HAMMER, 2001) e os demais testes com o programa Statistica 7.1. Análises inferenciais foram consideradas significativas em $\mathrm{p} \leq 0,05$.

\section{Resultados}

Foram coletados 1.320 espécimes distribuídos em cinco ordens, 22 famílias e 56 espécies, com biomassa total de $689 \mathrm{~kg}$. No período diurno foram amostrados 673 indivíduos, quatro ordens, 18 famílias e 47 espécies com biomassa total de $471 \mathrm{~kg}$ e, no período noturno, um total de 647 espécimes, cinco ordens, 19 famílias e 45 espécies, que apresentaram uma biomassa total de $218 \mathrm{~kg}$.

Diferenças foram encontradas para a abundância $(\mathrm{F}=18,51 ; \mathrm{p}<0,05)$ e biomassa $(\mathrm{F}=21,52 ; \mathrm{p}<0,05)$ 
entre as ordens de peixes amostradas. Os Characiformes capturados no período diurno $(\mathrm{p}=0,00002)$ e noturno $(\mathrm{p}=0,00001)$ apresentaram abundância superior aos Siluriformes coletados, respectivamente, em ambos os períodos (Figura 3). Os Characiformes capturados no período diurno $(\mathrm{p}=0,00001)$ e noturno $(\mathrm{p}=0,00001)$ apresentaram biomassa superior aos Siluriformes coletados, respectivamente, em ambos os períodos (Figura 4).

FIGURA 3: Abundância (média \pm erro padrão) das ordens dos peixes coletados no periodo diurno ( $\square$ ) e noturno ( $(\circ)$ em um treccho do rio Machado/RO. $\mathrm{CH}=$ Characiformes, $\mathrm{SI}=$ Siluriformes, $\mathrm{PE}=$ Perciformes, $\mathrm{CL}=$ Clupeiformes e GY = Gymnotiformes.

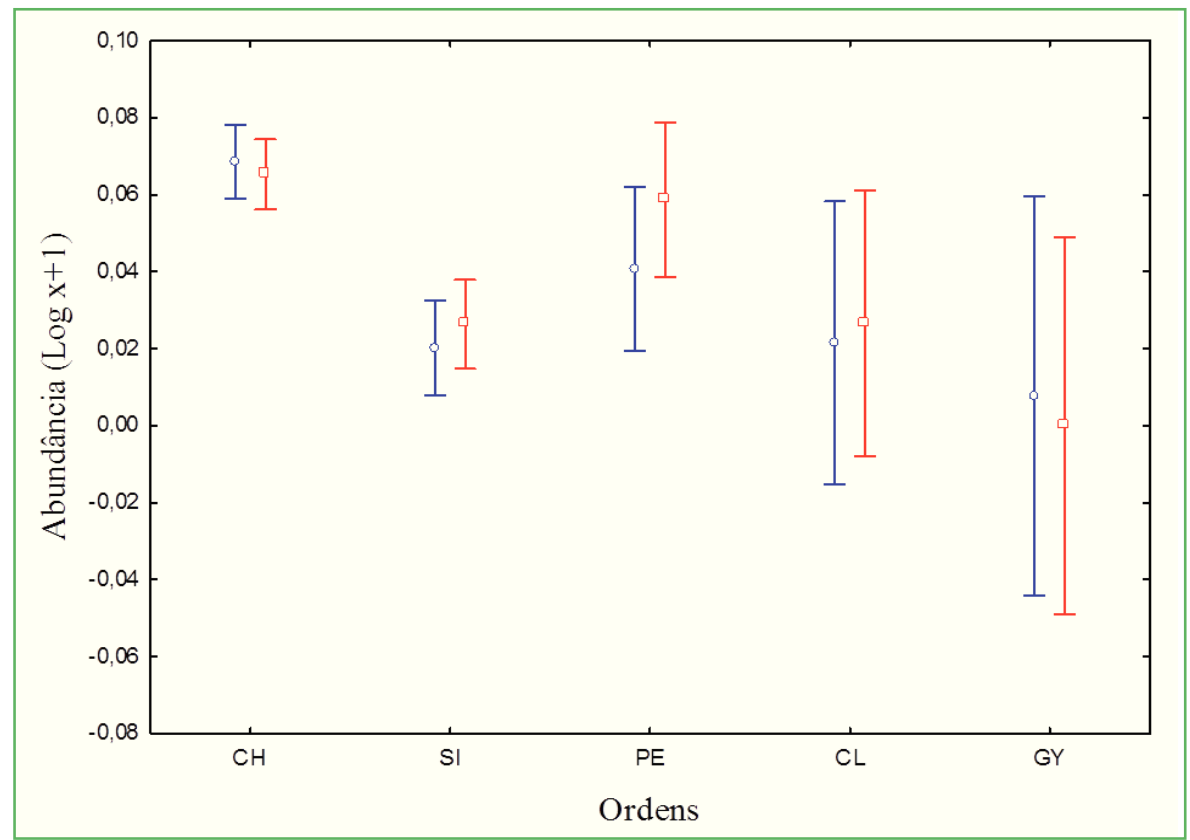

FIGURA 4: Biomassa (média \pm erro padrão) das ordens dos peixes coletados no periodo diurno ( $\square$ ) e noturno ( $\circ$ ) em um trecho do rio Machado/RO. $\mathrm{CH}=$ Characiformes, $\mathrm{SI}=$ Siluriformes, $\mathrm{PE}=$ Perciformes, $\mathrm{CL}=$ Clupeiformes e $\mathrm{GY}=$ Gymnotiformes.

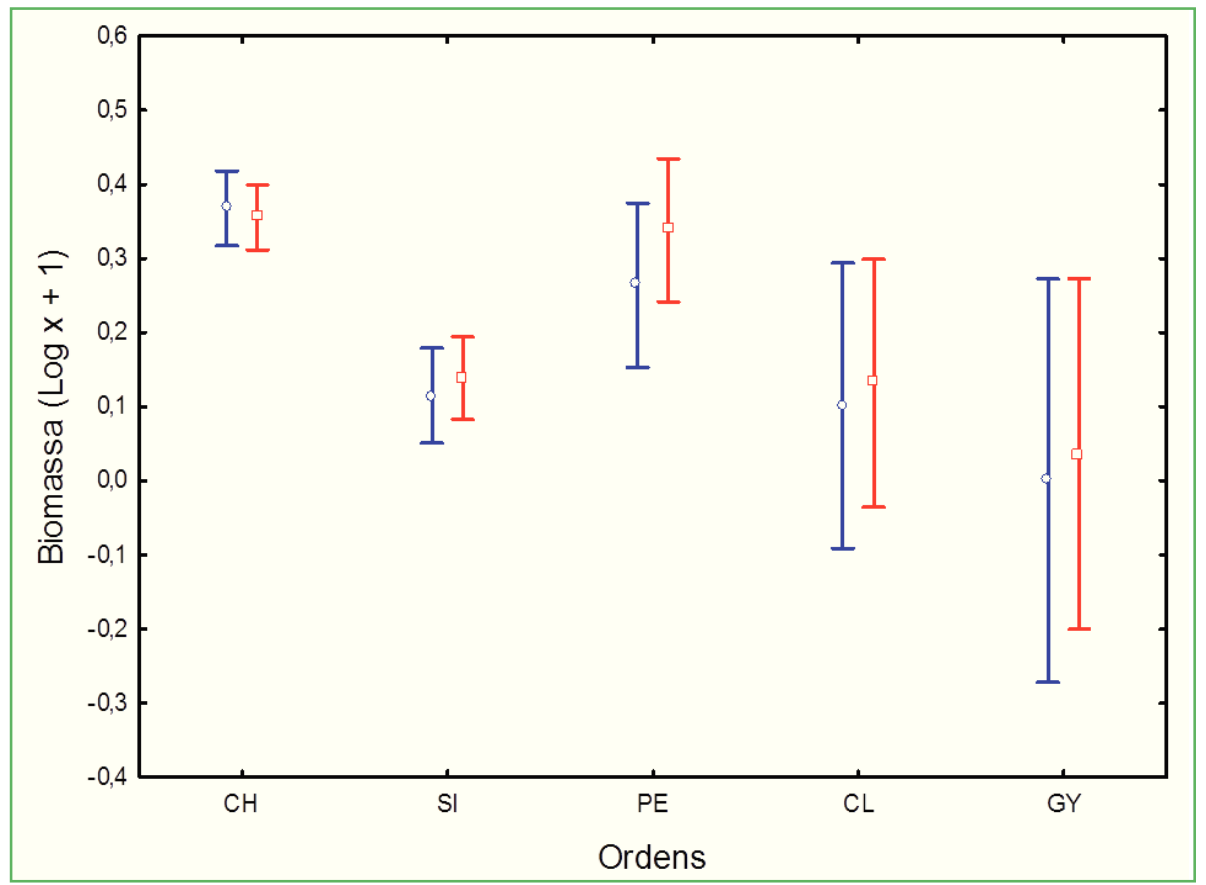


As espécies Serrasalmus rhombeus (Linnaeus, 1766), Plagioscion squamosissimus (Heckel, 1840) e Myleus torquatus (Kner, 1858) foram as mais representativas em abundância no período diurno. Serrasalmus rhombeus (Linnaeus, 1766) e M. torquatus foram as mais abundantes no período noturno. Para o período diurno as espécies $P$. squamosissimus, $M$. torquatus e $S$. rhombeus apresentaram maior biomassa, já no período noturno $P$. squamosissimus, $M$. torquatus e Hydrolicus scomberoides foram as mais representativas em biomassa (Tabela 2).

O EMnM das amostras com base na abundância e biomassa não indicou separação entre os períodos diurno e noturno nos eixos 1 e 2 retidos para interpretação $\left(\right.$ Stress $_{\text {Abundância }}=0,25 ;$ Stress $\left._{\text {Biomassa }}=0,24\right)($ Figuras 5 e 6$)$.

TABELA 2: Dados de abundância numérica $(\mathrm{N})$ e biomassa $(\mathrm{kg})$ das espécies coletados no período diurno e noturno no trecho médio do rio Machado - $\mathrm{RO} /$ Brasil.

\begin{tabular}{|c|c|c|c|c|}
\hline \multirow{2}{*}{ Ordem, família e espécies } & \multicolumn{2}{|c|}{ Diurno } & \multicolumn{2}{|c|}{ Noturno } \\
\hline & $\mathbf{N}$ & kg & $\mathbf{N}$ & kg \\
\hline \multicolumn{5}{|l|}{ Clupeiformes } \\
\hline \multicolumn{5}{|l|}{ Pristigasteridae } \\
\hline Pellona flavipinnis (Valenciennes, 1837) & 13 & 1,6 & 4 & 0,2 \\
\hline \multicolumn{5}{|l|}{ Engraulidade } \\
\hline Lycengraulis batesii (Günther, 1868) & 1 & 0,0 & 0 & 0,0 \\
\hline \multicolumn{5}{|l|}{ Characiformes } \\
\hline \multicolumn{5}{|l|}{ Acestrorhynchidae } \\
\hline Acestrorhynchus falcatus (Bloch, 1794) & 12 & 1,7 & 28 & 1,7 \\
\hline \multicolumn{5}{|l|}{ Anostomidae } \\
\hline Laemolyta taeniata (Kner, 1858) & 3 & 0,2 & 0 & 0,0 \\
\hline Leporinus agassizii Steindachner, 1876 & 0 & 0,0 & 1 & 0,0 \\
\hline Leporinus fasciatus (Bloch, 1794) & 4 & 0,8 & 16 & 3,6 \\
\hline Leporinus friderici (Bloch, 1794) & 4 & 0,6 & 7 & 0,7 \\
\hline \multicolumn{5}{|l|}{ Characidae } \\
\hline Astyanax bimaculatus (Linnaeus, 1758) & 18 & 0,1 & 1 & 0,0 \\
\hline Brycon pesu Müller Troschel, 1845 & 1 & 0,5 & 4 & 0,5 \\
\hline Bryconops caudomaculatus (Günther, 1864) & 3 & 0,0 & 9 & 0,1 \\
\hline Chalceus guaporensis Zanata \& Toledo-Piza, 2004 & 4 & 0,2 & 1 & 0,0 \\
\hline Charax gibbosus (Linnaeus, 1758) & 5 & 0,1 & 2 & 0,2 \\
\hline Colossoma macropomum (Cuvier, 1816) & 3 & 9,1 & 1 & 2,6 \\
\hline Moenkausia sp. & 3 & 0,2 & 10 & 0,1 \\
\hline Moenkhausia lepidura (Kner, 1858) & 1 & 0,0 & 0 & 0,0 \\
\hline Myleus torquatus (Kner, 1858) & 86 & 87,0 & 49 & 36,9 \\
\hline Roeboides affinis (Günther, 1868) & 0 & 0,0 & 16 & 0,2 \\
\hline Serrasalmus rhombeus (Linnaeus, 1766) & 116 & 34,2 & 154 & 17,7 \\
\hline Triportheus elongatus (Günther, 1864) & 14 & 1,3 & 1 & 0,2 \\
\hline Triportheus albus Cope, 1872 & 6 & 0,4 & 12 & 0,7 \\
\hline \multicolumn{5}{|l|}{ Chilodontidae } \\
\hline Caenotropus labyrinthicus (Kner, 1858) & 1 & 0,0 & 0 & 0,0 \\
\hline \multicolumn{5}{|l|}{ Ctenoluciidae } \\
\hline Boulengerella cuvieri (Spix \& Agassiz, 1829) & 22 & 32,9 & 10 & 8,3 \\
\hline \multicolumn{5}{|l|}{ Curimatidae } \\
\hline Curimata inornata Vari, 1989 & 19 & 0,7 & 43 & 1,5 \\
\hline Cyphocharax notatus (Steindachner, 1908) & 0 & 0,0 & 2 & 0,1 \\
\hline
\end{tabular}


Potamorhina latior (Spix \& Agassiz, 1829)

Psectrogaster amazonica Eigenmann \& Eigenmann, 1889

Cynodontidae

Hydrolycus scomberoides (Cuvier, 1819)

Rhaphiodon vulpinus Agassiz, 1829

Erythrinidae

Hoplias malabaricus (Bloch, 1794)

Hemiodontidae

Hemiodus unimaculatus (Bloch, 1794)

Prochilodontidae

Prochilodus nigricans Spix \& Agassiz, 1829

Siluriformes

Aspredinidae

Bunocephalus sp.

Loricariidae

Hypostomus plecostomus (Linnaeus, 1758)

Hypostomus sp.

Squaliforma emarginata (Valenciennes, 1840)

Panaque sp.

Sturisoma rostratum (Spix \& Agassiz, 1829)

Auchenipteridae

Ageneiosus inermis (Linnaeus, 1766)

Auchenipterus ambyiacus Fowler, 1915

Doradidae

Acanthodoras spinosissimus (Eigenmann \& Eigenmann, 1888)

Oxydoras niger (Valenciennes, 1821)

Platydoras costatus (Linnaeus, 1758)

Cetopsidae

Cetopsis candiru Spix \& Agassiz, 1829

Heptapteridae

Rhamdia quelen (Quoy \& Gaimard, 1824)

Pimelodidae

Hemisorubim platyrhynchos (Valenciennes, 1840)

Pimelodus blochii Valenciennes, 1840

Pseudoplatystoma tigrinum (Valenciennes, 1840)

Sorubim lima (Bloch \& Schneider, 1801)

Zungaro zungaro (Humboldt, 1821)

Gymnotiformes

Sternopygidae

Sternopygus macrurus (Bloch \& Schneider, 1801)

Perciformes

\section{Cichlidae}

Aequidens tetramerus (Heckel, 1840)

Astronotus ocellatus (Agassiz, 1831)

Cichla monoculus Spix \& Agassiz, 1831

Geophagus proximus (Castelnau, 1855)

Satanoperca jurupari (Heckel, 1840)

Scianidae

Plagioscion squamosissimus (Heckel, 1840)

$\begin{array}{cccc}13 & 0,9 & 28 & 0,8 \\ 4 & 0,0 & 2 & 0,1 \\ & & & \\ 34 & 30,9 & 34 & 33,1 \\ 10 & 11,6 & 13 & 8,5 \\ & & & \\ 0 & 0,0 & 1 & 0,0 \\ 2 & 0,2 & 1 & 0,6 \\ 55 & 28,7 & 69 & 27,2\end{array}$

0

0,0

0,0

18

17

2,3

24

2,0

2,0

0,5

3

0,4

0,2

0,0

0,2

0,0

0,0

1

22

2,0

0,0

1

0,0

0,7

0

0,0

0,2

1

6,0

0,0

13

2,2

0,3

0

0,0

0,1

2

0,3

0,0

5

1,8

3,6

6

1

4

2

0,5

1,2

$6,0 \quad 0 \quad 0,0$

$0,5 \quad 0 \quad 0,0$

$\begin{array}{lll}2,7 & 1 & 4,9\end{array}$

0

0,0

0,0

0

0,0

0,1

0,1

2

4,6

0,0

5

0,8

0,0

12

4

0,3

0,2

0,1

94

191,8

54,1 
FIGURA 5: Gráfico de EMnM para os períodos amostrados diurno ( $\square$ ) e noturno (०), baseado na abundância das espécies.

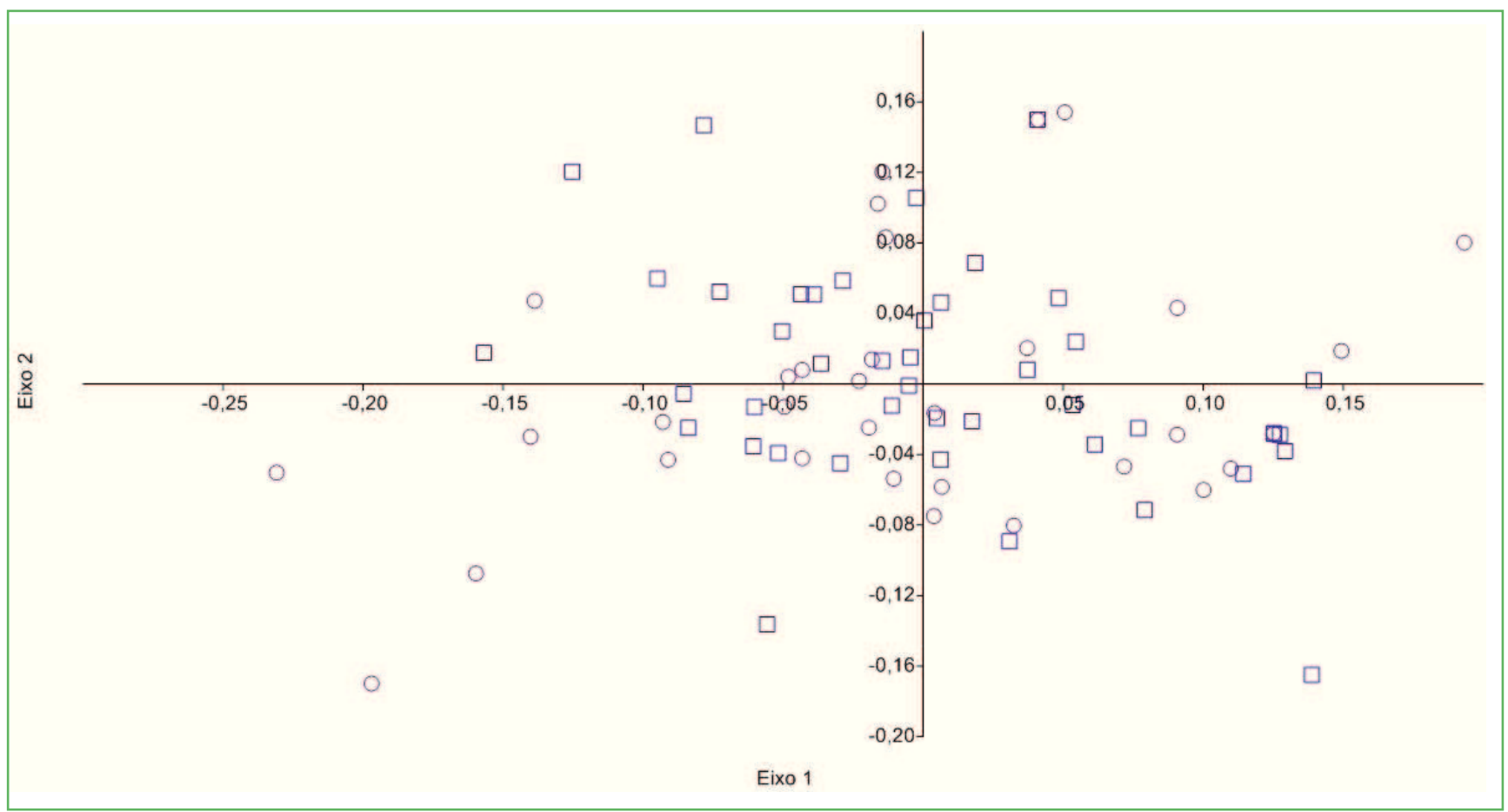

FIGURA 6: Gráfico de EMnM para os períodos amostrados diurno ( $\square$ ) e noturno (०), baseado na biomassa das espécies.

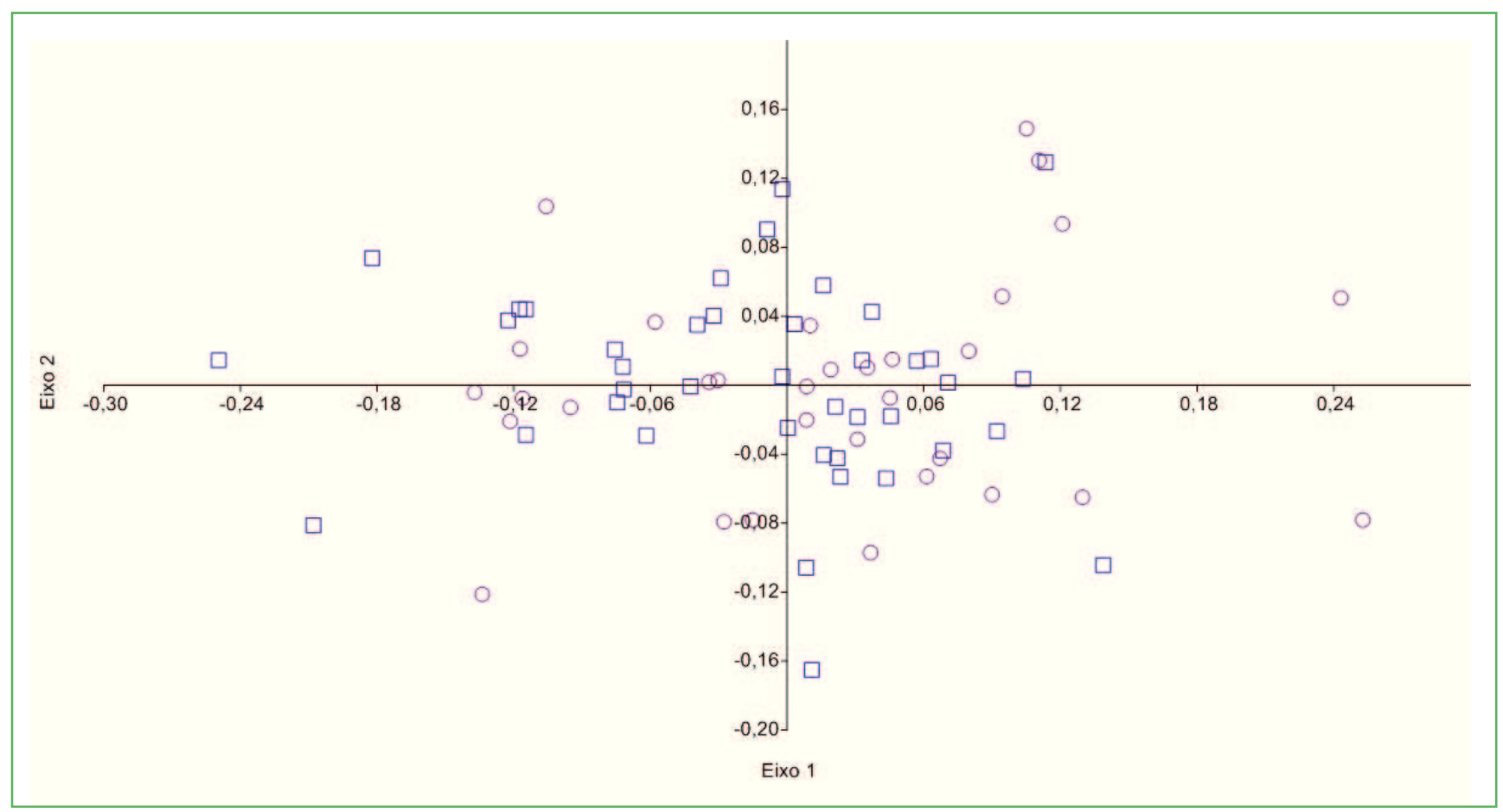


Não foram encontradas diferenças significativas para riqueza de espécies $(\mathrm{U}=718,0 ; \mathrm{p}=0,05)$, abundância $(\mathrm{t}=-0,66 ; \mathrm{p}=0,50)$ e biomassa $(\mathrm{U}=594,0$; $\mathrm{p}=0,17)$ entre os períodos diurno e noturno. Um total de $12(21 \%)$ espécies foi exclusivo do período diurno e oito $(14 \%)$ do período noturno.

\section{Discussão}

Sendo este caracterizado pela dominância de Characiformes (43\%), Siluriformes (36\%) e Gymnotiformes (3\%) representando aproximadamente $82 \%$ da ictiofauna amazônica (LOWE-MCCONNELL, 1999). Esta dominância é bem reportada para diversos rios na região Neotropical (SABINO; ZUANON, 1998; CASTRO, 1999; LOWE-MCCONNELL, 1999; POUILLY et al., 2004; COSTA; FREITAS, 2013).

Entre os peixes de água doce tropicais, Siluriformes e Gymnotiformes são geralmente descritos como pertencentes a ictiofauna noturna ou crepuscular, bem como alguns grupos marinhos (ex. Serranídeos e lutjanídeos). Por outro lado, os Characiformes e ciclídeos são predominantemente diurnos, como a maioria dos peixes recifais (ex. Chaedontídae, Pomacentridae, Labridae, Acanthuridae, Balistidae, Tetraodontidae, Diodontidae e predadores, como Synodontidae, Aulostomidae, Fistulariidae, Belonidae e Sphyraenidae) (LOWE-MCCONNELL, 1964; 1987; VOLPATO; TRAJANO, 2006). No entanto, muitas exceções são encontradas, apontando uma notória plasticidade ecológica dos peixes teleósteos. Como exemplo, mesmo entre os Siluriformes a atividade diurna foi relatada para espécies do gênero Trichomycterus (Trichomycteridae) e Ancistrus (Loricariidae) que geralmente são noturnos (BUCK; SAZIMA, 1995; CASATTI; CASTRO, 1998). A alimentação noturna parece incomum para pequenos loricarídeos (VOLPATO; TRAJANO, 2006), todavia, Sazima et al. (2000) descreveram a ocorrência de forrageamento crepuscular e noturno para Scoloplax empousa (Scoloplacidae).

Assim como em nossas pesquisas, Arrington e Winemiller (2003) apontam os Characiformes como grupo dominante em ambos os períodos, compondo $80 \%$ e $97 \%$ da abundância dos periodos diurno e noturno, respectivamente. Arrington e Winemiller (2003) e Shand (1997) descrevem que os Characiformes coletados exclusivamente em amostras noturnas, pertencem a espécies com corpo e olhos grandes, que presumivelmente aumentam a acuidade visual em ambientes de luz limitada. Atualmente, este atributo é descrito como uma adaptação à procura de alimentos em águas profundas, onde a luz é limitada diurnamente (STEWART et al., 2002) ou na captura de alimentos em águas rasas com luminosidade reduzida noturnamente, ou ambos (ARRINGTON; WINEMILLER, 2003).

A predominância de Characiformes em ambos os períodos para nossa pesquisa, é dada pela elevada representatividade da espécie $S$. rhombeus. Volpato e Trajano (2006) reportam que espécies carnívoras, como piranhas (Characidae) são ativas diurnamente, como esperado para espécies de Characiformes. Contudo, estudos de Sazima e Machado (1990) apontam que indivíduos de maior porte de Serrasalmus marginatus e S. spilopleura apresentaram atividade alimentar até o início da noite, e indivíduos de médio e grande porte de Pygocentrus nattereri forragearam principalmente na madrugada e próximo das $22 \mathrm{~h}$.

Rodríguez e Lewis (1997) propuseram que as assembleias de peixes de lagos inundados pelo rio Orinoco, Venezuela, são estruturadas pelo modelo Piscivoria-transparência-morfometria (PTM), que enfatiza a interação entre transparência do lago e as propriedades de cada espécie, em particular com as habilidades sensoriais e de forrageamento. O modelo PTM prevê que ao longo de gradientes espaciais ou temporais de declínio da transparência da água, a abundância de peixes diurnos, que utilizam principalmente a visão para a realização de suas atividades, diminui relativamente com relação aos peixes com adaptações à baixa visibilidade (RODRÍGUEZ; LEWIS, 1997).

Que corrobora como os estudos realizados em lagos no rio Araguaia/Tocantins, que apontam a diminuição da abundância de Characiformes e aumento de Siluriformes e ciclídeos em um gradiente de redução de transparência, não sendo encontrada variação na abundância de Clupeiformes e Scianidae para a variável analisada (TEJERINA-GARRO et al., 
1998). Embora os Clupeiformes sejam peixes visuais, estes podem reduzir sua dependência de visualização subaquática através de adaptações para a vida próxima à superfície da água, como a presença de escamas prateadas, corpo lateralmente comprimido, mandíbulas proeminentes e perfil dorsal-achatado (MARSHALL, 1971; MOYLE; CECH, 1996), já P. squamosissimus (Scianidae) está adaptado a alimentação próximo ou no fundo do ambiente, em condições de elevada turdidez, apresentando população de baixas densidades em águas claras (RODRÍGUEZ; LEWIS, 1997).

Desta forma, nós apontamos que como o rio estudado apresenta água clara (GOULDING, 1980; GOULDING et al., 2003), ou seja, a penetração de luz na coluna d'água não é interrompida por elevada carga de sedimento (água branca) ou a taxa de luminosidade é reduzida por conta da coloração escura oriunda da presença de ácidos húmicos e fúlvicos (água preta), a presença de uma fase clara e uma fase escura sobre a assembleia de peixes é evidente, o que possivelmente promoveria a alternância da composição ictiofaunística (Ordens) entre os períodos estudados. Todavia, em ambientes estruturalmente complexos, como o rio Machado (composto por corredeiras, pedrais, além de troncos e galhadas advindas da floresta marginal) possivelmente a eficiência dos aparelhos de coleta dos exemplares foi menor quando comparado a ambientes com menor complexidade estrutural, não sendo detectados períodos distintos de atividades para as espécies de peixes. Apontamos também, que hábitats com elevada complexidade estrutural apresentam menor variação na disponibilidade de recursos (alimento, sítios de reprodução, refúgio, etc), que resulta em menor movimentação dos peixes entre áreas marginais e águas abertas (PELICICE et al., 2005; WILLIS et al., 2005) promovendo ausência de diferença na abundância e composição de espécies entre os períodos do dia.

A maior representatividade das espécies $S$. rhombeus, M. torquatus e P. squamosissimus (Characiformes e Perciformes) no período diurno, e de $S$. rhombeus e $M$. torquatus no período noturno não corrobora os estudos de Fink e Fink (1979). Estes autores descrevem que a composição das assembleias de peixes pode variar de acordo com o ambiente e o período de atividade, apontando que Characiformes, Clupeiformes e Perciformes, que são peixes orientados visualmente e presentes em ambientes de águas mais transparentes, apresentam hábitos diurnos. Enquanto peixes orientados por estímulos químicos, tácteis ou elétricos como Siluriformes e Gymnotiformes, são encontrados principalmente em ambientes de águas mais turvas e de hábitos noturnos (LOWE-MCCONNEL, 1999).

Contudo, é possível a ocorrência de exceções ao padrão diurno/noturno de composição de espécies. A elevada representatividade da família Scianidae ( $P$. squamosissimus) em ambos os períodos, relaciona-se principalmente ao ritmo alimentar diário da espécie (HAHN et al., 1999). Barthem (1987) relatou que P. squamosissimus se caracterizou por um hábito predominantemente noturno: sua atividade se iniciou praticamente no começo da tarde, depois das $15 \mathrm{~h}$, e terminou entre $7 \mathrm{~h}$ e $9 \mathrm{~h}$. Em vista desses resultados, deduzimos que $P$. squamosissimus apresenta a mesma periodicidade alimentar quando se consideram distintos ambientes e horários. Esse fato pode estar relacionado ao comportamento diferenciado das presas (GLOVA et al., 1987) ou exploração de itens alimentares distintos ao longo do dia. Segundo estudo de Costa et al. (2009), referente ao espectro alimentar e variação sazonal da dieta de P. squamosissimus na lagoa do Piató (RN), os horários de atividade da espécie foram marcados por diferenças no tipo de alimento consumido, onde o item camarão predominou nas horas de menor luminosidade e o item insetos nas horas mais claras.

A utilização do ambiente em ambos os períodos do dia por uma dada espécie, também pode ser realizada através da segregação no tamanho dos indivíduos (SAZIMA; MACHADO, 1990). Todavia, para nossos resultados a maioria $(\mathrm{n}=10 ; 60 \%)$ dos juvenis $(<40 \mathrm{~cm})$ e adultos $(\mathrm{n}=45 ; 69 \%)$ de $P$. squamosissimus foram coletados no período noturno, reforçando a hipótese de que a elevada presença da espécie, em ambos os períodos, esta ligada a exploração de recursos alimentares. Pesquisas de Duarte et al. (2010), em praias do rio Purus (AM) descrevem a ocorrência, com baixa abundância, de $M$. torquatus em ambos os períodos dia. Membros da sub-familia Serrasalminae, mais especificamente 
do gênero Mylossoma e Myleus, apresentam hábito alimentar herbívoro sendo provavelmente tal recurso explorado em todos os horários do dia.

Nossa análise exploratória não identificou segregação entre os períodos diurno e noturno baseado na abundância e biomassa das espécies. Matthews (1998) descreve que em grandes rios e lagos, há poucos limites físicos observáveis para as atividades diárias da maioria dos peixes. Muitas espécies de peixes de lagos apresentam um padrão diário de movimentação da região litoral para águas abertas e vice-versa (MATTHEWS, 1998; COSTA et al., 2011) e assim são, muitas vezes, membros de diferentes assembleias espacialmente definidas (MATTHEWS, 1998). Tal separação espacial diminui a detecção de diferenças nictimerais quando analisadas assembleias de peixes de hábitats específicos. A ausência de segregação entre os períodos analisados esta relacionada com a distribuição praticamente equitativa de um elevado número de indivíduos e biomassa de alguns táxons (ex. Characiformes, Characidae e Perciformes, Scianidae) que apresentam atividade em ambos os períodos (BARTHEM, 1987; SAZIMA; MACHADO, 1990; HAHN et al., 1999; COSTA et al., 2009; DUARTE et al., 2010).

Diferenças na abundância e biomassa das espécies ativas no período diurno e noturno não foram observadas. Resultados similares foram encontrados por alguns autores (PELICICE et al., 2005; COSTA; FREITAS, 2010; SACCOL-PEREIRA; FIALHO, 2010). Estudos de Willis et al. (2005) descrevem baixa taxa de substituição de espécies entre os períodos diurno e noturno, principalmente em ambientes estruturalmente complexos no período da seca. Peixes apresentam menor movimentação para as áreas marginais (refúgio) no período noturno em ambientes com maior complexidade estrutural (WILLIS et al., 2005), além de que hábitats mais complexos apresentam maior estabilidade de recursos, que resulta em menor movimentação entre áreas marginais e águas abertas para forrageamento durante o dia (PELICICE et al., 2005; WILLIS et al., 2005), que corrobora com as condições estruturais de nossa área de estudo, composta principalmente por rochas isoladas, pedrais, troncos e galhos de árvores.
Segundo Correa et al. (2008), malhadeiras capturam peixes que estão ativamente em movimento, desta forma este aparato reflete principalmente as alterações na atividade nictemeral da ictiofauna, em vez de mudanças na abundância das espécies em seu hábitat. Alguns estudos destacam a importância da periodicidade circadiana na atividade dos peixes (GAUDREAU; BOISCLAIR, 1998; JACOBSEN; PERROW, 1998; AGOSTINHO et al., 2005), onde os movimentos destes podem promover mudanças na composição das assembleias de peixes, bem como uma aumento da pressão de predação nos períodos crepusculares. No entanto, não observamos efeitos significativos em relação a periodicidade diurna.

Nossos resultados indicam a ausência de segregação nictemeral no biótopo estudado com base na abundância e biomassa, apontando principalmente que as espécies pertencentes ao grupo dos Characiformes permanecem ativas no ambiente estudado em todo o período do dia, sendo tal organização temporal provavelmente associada à exploração de recursos alimentares no ambiente. Contudo, também é evidenciada a contribuição em abundância de determinadas ordens (Perciformes e Siluriformes) na utilização do ambiente em períodos distintos aos citados pela literatura, sendo tal padrão provavelmente relacionado à elevada disponibilidade de hábitats complexos que favorece uma maior aquisição de recursos.

Apesar da não existência de diferenças nos descritores ecológicos supracitados para ambos os períodos analisados, apontamos que amostragens nos períodos diurno e noturno são necessárias. A ocorrência de um pequeno número de espécies raras e exclusivas do período diurno $(\mathrm{S}=12 ; 21 \%)$ e noturno $(\mathrm{S}=8$; $14 \%$ ), é relevante para a completude do conhecimento ictiofaunístico regional, principalmente para a realização de inventários taxonômicos que antecedem a instalação de grandes empreendimentos (ex. represas, fábricas, áreas de cultivo e pecuária) ou áreas de proteção ambiental.

\section{Agradecimentos}

Ao Instituto Chico Mendes de Conservação da Biodiversidade, pelo apoio logístico e financeiro, ao 
Mestre Piatã Marques, da University of Victoria, pelo auxilio na confecção do abstract, e ao Laboratório de Ictiologia e Pesca da Universidade Federal de Rondônia, pela ajuda no tombamento e identificação taxonômica do material.

\section{Referências}

AGOSTINHO,A.A.; THOMAZ, S. M.; GOMES, L.C. Conservation of the biodiversity of Brazil's inland water. Conservation Biology, San Francisco, v. 19, n. 3, p. 646-652, 2005.

ARRINGTON, D. A.; WINEMILLER, K. O. Diel changeover in sandbank fish assemblages in a neotropical floodplain river. Journal of Fish Biology, Malden, v. 63, p. 442-459, 2003.

BARTHEM, R. B. Uso de redes de espera no estudo de ritmos circadianos de algumas espécies de peixes nos lagos de várzea do rio Solimões. Revista Brasileira de Zoologia, Curitiba, v. 3, n. 7, p. 409-422, 1987.

BOUJARD, T.; LEATHERLAND, J. F. Circadian rhythms and feeding time in fishes. Environmental Biology Fishes, New York, v. 35, p. 109-131, 1992.

BUCK, S. M. C.; SAZIMA, I. An assemblage of mailed catfishes (Loricariidae) in southeastern Brazil: distribution, activity, and feeding. Ichthyology Explore Freshwaters, Cornol, v. 6, n. 4, p. 325-332, 1995.

CASATTI, L.; CASTRO, R. M. C. A fish community of the São Francisco River headwaters riffes, southeastern Brazil. Ichthyology Explore Freshwaters, Cornol, v. 9, n. 3, p. 229-242, 1998.

CASTRO, R. M. C. Evolução da ictiofauna de riachos sulamericanos: padrões gerais e possíveis processos casuais. In: CARAMASCHI, E. P., MAZZONI, R.; PERES-NETO, R. (Ed.). Ecologia de peixes de riachos. 1. ed. Rio de Janeiro: Série Oecologia Brasiliensis, 1999. p. 139-155.

CARVAlHO, L. N.; ZUANON, J. A. S.; SAZIMA, I. Natural history of Amazon fishes. 2007. Disponivel em: <http://www. eolss.net>. Acesso em: 18 jun. 2014.

CORREA, S. B.; CRAMPTON, W. G. R.; CHAPMAN, L. J.; ALBERT, J. S. A comparison of flooded forest and floating meadow fish assemblages in an upper Amazon floodplain. Journal of Fish Biology, Malden, v. 72, n. 3, p. 629-644, 2008.

COSTA, I. D.; FREITAS, C. E. C. Variação nictemeral na composição e abundância da ictiofauna em um trecho do rio Urucu - Coari/Amazonas/Brasil. Revista Colombiana Ciencia Animal, Sincelejo, v. 2, n. 2, p. 355-364, 2010.

COSTA, I. D.; FREITAS, C. E. C. Trophic ecology of the ichthyofauna of a stretch of the Urucu River (Coari, Amazonas, Brazil). Acta Limnologica Brasiliensia, Rio Claro, v. 25, n. 25, p. 54-67, 2013.

COSTA, I. D.; ROCHA, A. C. P. V.; LIMA, M. L. C.; ZUANON, J. A. S. Composição e abundância de peixes da interface entre as águas abertas e bancos de macrófitas e sua dinâmica nos períodos de crepúsculos matutino e vespertino, no lago Catalão, Amazonas, Brasil. Biotemas, Florianópolis, v. 24, n. 2, p. 97-101, 2011.

COSTA, S. A. G. L.; PERETTI, D.; JÚNIOR, J. P. M. P.; FERNANDES, M. A.; JÚNIOR, A. M. G. Espectro alimentar e variação sazonal da dieta de Plagioscion squamosissimus (Heckel, 1840) (Osteichthyes, Sciaenidae) na lagoa do Piató, Assu, estado do Rio Grande do Norte, Brasil. Acta Scientiarum, Maringá, v. 31, n. 3, p. 285-292, 2009.

CPRM - COMPANHIA DE PESQUISA DE RECURSOS MINERAIS. Mapa geológico 1:2.500.000. Porto Velho: Diretoria de Serviços Geográficos, 2012. 30 p.

DUARTE, C.; RAPP PY-DANIEL, L. H.; DEUS, C. P. Fish assemblages in two sandy beaches in lower Purus River, Amazonas, Brazil. Iheringia, Série Zoologia, Porto Alegre, v. 100, n. 4, p. 319-328, 2010.

DUARTE, C.; SOUZA, V. S.; NUNES, C. O. Variação nictemeral na composição da ictiofauna no lago Catalão (confluência dos rios Solimões e Negro). Amazon Science, Manaus, v. 1, n. 1, p. 18-27, 2012.

FERNANDES, L. C.; GUIMARÃES, S. C. P. Atlas geoambiental de Rondônia. Porto Velho: SEDAM, 2002. 151 p.

FERREIRA, E. J. G.; ZUANON, J. A. S.; SANTOS, G. M. Peixes comerciais do Médio Amazonas: região de Santarém, Pará. Brasília: IBAMA, 1998. 75 p.

FINK, W. I.; FINK, S. V. A Amazônia Central e seus Peixes. Acta Amazonica, Manaus, v. 8, p. 19-42, 1979.

GAELZER, L. R.; MACHADO, G. R.; NOGUCHI, R. C. Peixes de praias arenosas. In: CREED, J. C.; PIRES, D. O.; FIGUEIREDO, M. A. O. (Ed.). Biodiversidade marinha da baía da Ilha Grande. 1 ed. Brasília: Série Biodiversidade, 2007. p. 320-416.

GAUDREAU, N.; BOISCLAIR, D. The influence of spatial heterogeneity on the study of fish horizontal daily migration. Fisheries Research, St John's, v. 35, n. 1-2, p. 65-73, 1998.

GLOVA, G. J.; SAGAR, P. M.; DOCHERTY, C. R. Diel feeding periodicity of torrestfish (Cheimarrichthys fosteri) in two braided rivers of Canterbury, New Zealand. New Zeland Journal of Marine and Freshwater Research, Nelson, v. 21, p. 555-561, 1987.

GOULDING, M. The fishes and the forest: explorations in Amazonian natural history. Berkey: University of California Press, 1980. 280 p.

GOUldinG, M.; BARTHEM. R.; FERREIRA. E. The Smithsonian Atlas of the Amazon. Washington: Smithsonian Institution, 2003. $232 \mathrm{p}$.

HAHN, N. S.; LOUREIRO, V. E.; DELARIVA, R. L. Atividade alimentar da curvina Plagioscion squamosissimus (Heckel, 1940) (Perciformes, Scianidae) no rio Paraná. Acta Scientiarum, Maringá, v. 21, n. 2, p. 309-314, 1999.

HAMMER, O. PAST: Paleontological Statistics Software Package for Education and Data Analysis. Palaeontologia Electronica, Oslo, v. 4, p. 9, 2001.

IBGE - INSTITUTO BRASILEIRO DE GEOGRAFIA E ESTATÍSTICA. Manual técnico da vegetação brasileira. Rio de Janeiro: IBGE, 1992. 92 p.

IIGO, M.; TABATA, M. Circadian rhythms of locomotor activity in the goldfish Carassius auratus. Physiology \& Behavior, Oxford, v. 60 , n. 3, p. 775-781, 1996.

JACOBSEN, L.; PERROW, M. R. Predation risk from piscivorous fish influencing the diel use of macrophytes by planktivorous fish 
in experimental ponds. Ecological Freshwater Fishes, Malden, v. 7, n. 2, p. 78-86, 1998.

KRUSCHE, A. V.; BALLESTER, M. V. R.; VICTORIA, R. L.; BERNARDES, M. C.; LEITE, N. K.; HANADA, L.; VICTORIA, D. C.; TOLEDO, A. M.; OMETTO, J. P.; MOREIRA, M. Z.; GOMES, B. M.; BOLSON, M. A.; NETO, S. G.; BONELLI, N.; DEEGAN, L.; NEILL, C.; THOMAS, S.; AUFDENKAMPE, A. K.; RICHEY, J. E. Efeitos das mudanças do uso da terra na biogeoquímica dos corpos d'água da bacia do rio Ji-Paraná, Rondônia. Acta Amazonica, Manaus, v. 35, n. 2, p. 197-205, 2005. LAMPRECHT, G.; WEBER, F. Spontaneous locomotion behaviour in cavernicolous animals: the regression of the endogenous circadian system. In: CAMACHO, A. I. (Ed.). The natural history of Biospeleology. 1. ed. Madrid: Monografias del Museo Nacional de Ciencias Naturales, 1992. p. 225-262.

LOWE-MCCONNELL, R. H. The fishes of the Rupununi savanna district of British Guiana, South America. Part 1. Ecological groupings of fish species and eVects of the seasonal cycle on the fish. Zoological Journal of the Linnean Society, London, v. 45, n. 304, p. 103-144, 1964.

LOWE-MCCONNELL, R. H. Ecological studies in tropical fish communities. New York: Press Syndicate of de University of Cambridge, 1987. 382 p.

LOWE-MCCONNELL, R. H. Estudos ecológicos em comunidades de peixes tropicais. São Paulo: EDUSP, 1999. 321 p.

MARSHALL, N. B. Explorations in the life of fishes. Cambridge: Harvard University Press, 1971. 204 p.

MATTHEWS, W. J. Patterns in freshwaters fish ecology. Oklhoma: University of Oklahoma Press, 1998. 756 p.

MAZZEO, N. L.; RODRÍGUEZ-GALLEGO, G.; KRUK, M.; MEERHOFF, J.; GORGA, G.; LACEROT, F.; QUINTANS, M.; LOUREIRO, D.; LARREA. F.; GARCÍA-RODRÍGUES, T. Effect of Egeria densa Planch. beds on a shallow lake without piscivorous fish. Hydrobiologia, Brussels, v. 506/509, p. 591-602, 2003.

MENEZES, N. A. Methods for assessing freshwater fish diversity. In: MENEZES, N. A.; BICUDO, C. E. M. (Ed.). Biodiversity in Brazil: a first approach. 1. ed. São Paulo: Conselho Nacional de Desenvolvimento Científico e Tecnológico, 1996. p. 289-295.

MMA - MINISTÉRIO DO MEIO AMBIENTE. Reserva Biológica do Jaru. Porto Velho: Ministério do Meio Ambiente/Instituto Chico Mendes de Conservação da Biodiversidade, 2010. 300 p.

MOYLE, P. B.; CECH, J. J. Jr. Fishes: an introduction to ichthyology. Englewood Cliffs: Prentice-Hall, 1996. 590 p.

NELSON, J. S. Fishes of the world. New Jersey: John Wiley and Sons, $1994.600 \mathrm{p}$.

NELSON, J. S. Fishes of the world. New Jersey: John Wiley and Sons, 2006. $601 \mathrm{p}$.

PELICICE, F. M.; AGOSTINHO, A. A.; THOMAZ, S. M. Fish assemblages associated with Egeria in a tropical reservoir: investigating the effects of plant biomass and diel period. Acta Oecologica, New York, v. 27, p. 9-16, 2005.
POUILLY, M.; YUNOKI, T.; ROSALES, C.; TORRES, L. Trophic structure of fish assemblages from Mamoré River floodplain lakes (Bolivia). Ecology Freshwater Fishes, Malden, v. 13, n. 4, p. 245257, 2004.

REIS, R. E.; KULLANDER, S, O.; FERRARIS, JR. Check list of the freshwater of South and Central America. Porto Alegre: EDIPUCRS, 2003. 729 p.

RODRÍGUEZ, M. A.; LEWIS, W. M. JR. Structure of fish assemblages along environmental gradients in floodplain lakes of the Orinoco River. Ecology Monography, Ithaca, v. 67, n. 1, p. 109-128, 1997.

SABINO, J.; ZUANON. J. A. S. A stream fish assemblage in Central Amazonia: distribution, activity patterns and feeding behavior. Ichthyology Explorer Freshwater, Cornol, v. 8, n. 3, p. 201-210, 1998.

SACCOL-PEREIRA, A.; FIALHO, C.B. Seasonal and diel variation in the fish assemblage of a Neotropical delta in southern Brazil. Iheringia, Série Zoologia, Porto Alegre, v. 100, n. 2, p. 169-178, 2010.

SÁNCHEZ-VÁZQUEZ， F. J.; MADRID， J. A.; IIGO, M.; TABATA, M. Demand feeding and locomotor circadian rhythms in the goldfish, Carassius auratus: dual and independent phasing. Physiology \& Behavior, Oxford, v. 60, n. 2, p. 665-674, 1996.

SANTOS, G. M.; FERREIRA, E. J. G.; ZUANON, J. A. S. Peixes comerciais de Manaus. Manaus: ProVárzea, 2006. 144 p.

SAZIMA, I.; MACHADO, F.A. Underwater observations of piranhas in western Brazil. Environmental Biology of Fishes, New York, v. 28, n. 1-4, p. 17-31, 1990.

SAZIMA, I.; MACHADO, F. A.; ZUANON, J. Natural history of Scoloplax empousa (Scoloplacidae), a minute spiny catfish from the Pantanal wetlands in western Brazil. Ichthyology Explore Freshwaters, Cornol, v. 11, n. 1, p. 89-95, 2000.

SHAND, J. Ontogenetic changes in retinal structure and visual acuity: A comparative study of coral-reef teleosts with differing post-settlement lifestyles. Environmental Biology of Fishes, New York, v. 49, n. 3, p. 307-322, 1997.

STEWART, D. J.; IBARRA, M.; BARRIGA-SALAZAR, R. Comparison of deep-river and adjacent sandy-beach fish assemblages in the Napo River Basin, Eastern Ecuador. Copeia, Lawrence, v. 2, n. 2, p. 33-343, 2002.

TEJERINA-GARRO, F. L.; FORTIN, R.; RODRÍGUEZ, M. A. Fish community structure in relation to environmental variation in floodplain lakes of the Araguaia River, Amazon Basin. Environmental Biology of Fishes, New York, v. 51, n. 4, p. 399410, 1998.

VOLPATO, G. L.; TRAJANO, E. Biological rhythms. In: VAL, L. A.; VAL, V. M. F. A.; RANDAL, D. J. (Ed.). Fish physiology. 1. ed. San Diego: Elsevier, 2006. p. 101-153.

WILLIS, S. C.; WINEMILLER, K. O.; LOPEZ-FERNANDEZ, H. Habitat structural complexity and morphological diversity of fish assemblages in a Neotropical floodplain river. Oecologia, Heidelberg, v. 142, p. 284-295, 2005. 